\title{
Desempeño económico y protesta ciudadana como detonantes de las caídas presidenciales: el caso ecuatoriano
}

\author{
Santiago Basabe-Serrano,* John Polga-Hecimovich**
}

Perfiles Latinoamericanos, 25(50)

2017 | pp. $129-153$

DOI: $10.18504 / \mathrm{pl} 2550-007-2017$

\begin{abstract}
Resumen
En este artículo argumentamos que las crisis económicas $\left(X_{1}\right)$ a las que siguen movilizaciones sociales en las calles $\left(X_{2}\right)$ explican las salidas anticipadas de presidentes $(Y)$. Luego de identificar los puntos de acuerdo y tensión hallados en los trabajos hasta ahora realizados, proponemos que el estudio de las caídas presidenciales puede hallar un espacio fructífero a partir del análisis de causalidad coyuntural múltiple. Mediante dos casos de inestabilidad presidencial ocurridos en Ecuador, este artículo sugiere que las discrepancias halladas en la literatura podrían ser zanjadas si se presta atención a enfoques teóricos más analíticos como el que aquí proponemos.
\end{abstract}

\begin{abstract}
In this article, we identify economic crises $\left(X_{1}\right)$ in the presence of social mobilization $\left(X_{2}\right)$ as jointly conditional causes of premature president exit $(Y)$. After identifying common points and tension in the literature, we propose that the study of presidential removal can benefit by incorporating a multiple conjunctural causation framework. Using two case studies of presidential instability in Ecuador, the article suggests that theoretical-empirical discrepancies in the existing literature can be resolved be incorporating more analytically rigorous approaches such as the one we identify.
\end{abstract}

Palabras clave: causalidad coyuntural múltiple, caídas presidenciales, Ecuador, desempeño económico, protesta ciudadana.

Keywords: Complex causality, presidential instability, Ecuador, economic crises, social mobilization.

* Doctor en Ciencia Política por la Universidad Nacional de San Martín, Buenos Aires-Argentina. Becario Georg Foster de la Fundación Alexander von Humboldt e investigador posdoctoral del Instituto Alemán de Estudios Globales y de Área (GIGA), Hamburgo, Alemania. Coordinador de la Maestría en Política Comparada y profesor titular del Departamento de Estudios Políticos de la Facultad Latinoamericana de Ciencias Sociales, Sede Ecuador | sbasabe@flacso.edu.ec

** Doctor en Ciencia Política por la Universidad de Pittsburgh. Profesor asistente del Departamento de Ciencia Política de la U. S. Naval Academy, Estados Unidos | jop42@pitt.edu 


\section{Introducción ${ }^{1}$}

—uego del retorno al régimen democrático, muchos países de América Latina afrontaron momentos de inestabilidad presidencial que en ocasiones desembocaron en la salida anticipada de los jefes de Estado. Para el estudio de dicho fenómeno político, la literatura especializada ha avanzado en dos frentes. De un lado, están los trabajos focalizados en observar cómo estas formas no convencionales de resolución de conflictos entre poderes del Estado permiten dar una salida política sin resquebrajar el régimen democrático (Mejía \& Polga-Hecimovich, 2011; Kim \& Bahry, 2008; Marsteintredet \& Berntzen, 2008). De otro, están las investigaciones que se concentran en identificar las variables que explican las salidas anticipadas de los presidentes (Kim, 2013; Pérez-Liñán, 2007, 2014; Hochstetler, 2006; Cheibub, 2002).

En el primer frente, la discusión teórica gira alrededor del argumento de Juan Linz (1990), quien plantea que el presidencialismo puede llevar a que las tensiones entre ejecutivo y legislativo alcancen un punto tal en el que la democracia se menoscaba. En ese escenario, se apunta a que las salidas anticipadas de los presidentes son formas ad hoc para mantener el régimen democrático a pesar de la inestabilidad generada. En el segundo frente, los debates teóricos proponen observar cómo las diferentes correlaciones de fuerzas políticas, los diseños institucionales o las variables del entorno económico o social inciden en la estabilidad presidencial.

Este artículo se vincula con el segundo grupo de trabajos investigativos, enfatizando en los desarrollados para América Latina. En esencia, nos proponemos identificar las distintas variables asumidas como explicativas de las caídas presidenciales para posteriormente retestearlas en dos acontecimientos emblemáticos de Ecuador, uno de los países más inestables de la región. El artículo se compone de cuatro partes. En la primera se revisan los principales hallazgos empíricos identificados en la literatura especializada. En la segunda, se propone un marco teórico en el que se argumenta que las crisis económicas a las que siguen protestas sociales en las calles constituyen las variables que, vinculadas entre sí, dan lugar a las caídas presidenciales. En la tercera, se testea dicha propuesta teórica recurriendo a dos casos de inestabilidad presidencial suscitados en Ecuador. La cuarta parte propone conclusiones y algunas recomendaciones de cara a futuras agendas de investigación.

1 Una versión previa fue presentada durante el Congreso Anual de la Midwest Political Science Association (MPSA), el 3 de abril de 2013. Los autores agradecen a Ezequiel González Ocantos y Kathryn Hochstetler por sus valiosos comentarios. 


\section{La inestabilidad presidencial: el estado de la literatura especializada}

El estudio de las caídas presidenciales fue uno de los temas que mayor espacio capturó en la agenda de investigación de América Latina entre los años noventa y la primera década del siglo xxi. Dicho fenómeno fue analizado desde diversas perspectivas teóricas y estrategias metodológicas, sin llegar a consensos en cuanto a los factores que mejor explican las salidas anticipadas de los presidentes. Así, aunque hay una serie de variables que se repite sistemáticamente en la investigación, no hay consenso sobre cuál de ellas es la que más influye en las caídas presidenciales.

Del conjunto de variables identificadas por la literatura especializada, un primer grupo son explicaciones esencialmente políticas y tienen que ver con las relaciones entre los poderes ejecutivo y legislativo y específicamente con las características de las coaliciones de gobierno. En este grupo se incluye también el apoyo electoral recibido tanto por el presidente como por el partido de gobierno en tanto posibles desencadenantes de las salidas anticipadas de los jefes de Estado. Un segundo conjunto de explicaciones señala que los desencadenantes de los casos de inestabilidad presidencial son variables relacionadas con las políticas y el desempeño económicos de los gobiernos. Finalmente, un tercer grupo argumenta que son variables del entorno social, como la protesta de la ciudadanía o los escándalos de corrupción, las que constituyen el "disparador" de la salida anticipada de los presidentes. A continuación discutimos las tres vertientes.

Coaliciones legislativas y apoyo electoral al partido en el gobierno y al presidente

Una de las ideas centrales de este tipo de explicaciones sostiene que la debilidad del apoyo legislativo al ejecutivo, observable sobre todo en coaliciones de gobierno poco eficientes, constituye la variable clave para entender las caídas presidenciales. Dentro de esta tradición se encuentran Carey (2003a) para el caso peruano, Weyland (1993) para el brasileño y Schamis (2002) para el argentino. Considerando un universo de países más amplio, también se sitúan aquí Carey (2003b), Valenzuela (2004), Hochstetler (2006) y Pérez-Liñán (2014). Para el caso de Ecuador, Mejía \& Polga-Hecimovich (2011) afirman que son los cambios constitucionales orientados a disminuir la disponibilidad y discrecionalidad del presidente en el manejo de recursos económicos lo que desincentiva la formación de coaliciones de gobierno duraderas. Como consecuencia, las probabilidades de inestabilidad política que desembocan en salida anticipada de los presidentes aumentarían. 
Relacionado con dicho argumento, algunos estudios de los sistemas parlamentarios señalan que la alta fragmentación del régimen de partidos se vincula con la mayor fragilidad política del ejecutivo y los posibles efectos que de allí se derivan en términos de transitoriedad presidencial (Kim \& Bahry, 2008). En ese plano, cuanto mayor sea el número de partidos representados en la legislatura disminuyen las probabilidades de formar coaliciones de gobierno y, por lo tanto, crecería la propensión a que se verifiquen caídas presidenciales. Un efecto intermedio de la alta fragmentación política es el incremento de la negociación entre presidentes y legisladores de cara a conseguir acuerdos políticos (Mainwaring \& Shugart, 1997; Jones, 1995; Mainwaring, 1993).

Otro argumento inscrito en las variables de naturaleza política señala que los presidentes con menor cantidad de asientos en la legislatura son más proclives a generar tensiones entre poderes del Estado a tal grado que la crisis se resuelve con la salida anticipada del ejecutivo (Negretto, 2006; Cox \& Morgenstern, 2002; Valenzuela, 2004). En conformidad con dicha explicación, en un estudio cuantitativo que abarca ejemplos de América Latina, Europa, Asia y África, Kim \& Bahry (2008) concluyen que esta variable es clave para explicar las caídas de líderes políticos. No obstante, Cheibub (2002) presenta hallazgos empíricos que cuestionan los ya citados. En efecto, una de las conclusiones a las que este último autor arriba, luego de analizar todas las democracias existentes entre 1946 y 1996, es que la interacción de gobiernos de minoría derivados de presidentes con bajo apoyo legislativo y tensas relaciones con el legislativo no afecta la sobrevivencia de los regímenes democráticos. Dada la relación entre la caída de los presidentes y el desplome del régimen democrático en el periodo que antecedió a los años noventa, una extensión de los hallazgos de Cheibub (2002) arroja dudas sobre la relación causal entre el bajo apoyo legislativo y la pugna de poderes respecto a la supervivencia de los gobiernos.

La ausencia del apoyo popular mayoritario en el momento de la elección constituye una explicación adicional a la caída de los presidentes. Dado que la tendencia "natural" del ciclo político es que los niveles de aceptación ciudadana de los presidentes desciendan a medida que transcurre el tiempo de su mandato, si al inicio de la gestión la votación obtenida no fue suficientemente sólida, en poco tiempo el respaldo popular será deficitario. En condiciones de debilidad política como la anotada, las probabilidades de que los presidentes salgan de sus cargos antes de concluir el periodo constitucional establecido son mayores. Kim \& Bahry (2008: p. 816) han planteado que tanto esta variable como la cantidad de asientos legislativos del presidente influyen en el aumento de las caídas presidenciales. No obstante, al incluir ambas variables en el modelo propuesto por las autoras, solo el apoyo popular al presidente en las elecciones mantiene valores significativos. 
Los desempeños deficientes de la economía son otra de las variables que la literatura ha citado como influyente en la salida anticipada de los presidentes (Kim \& Bahry, 2008; Hochstetler, 2006; Valenzuela, 2004; Pérez-Liñán, 2003). $\mathrm{Al}$ respecto, Haggard \& Kaufman (1995) han argumentado que la presencia de declives económicos seguidos por medidas de emergencia o programas de austeridad incrementan el descontento de la ciudadanía, lo que resulta en una mayor fragilidad presidencial. Sin embargo, también existen hallazgos opuestos. Con abundante evidencia empírica, Cheibub (2002) afirma que las condiciones económicas no tienen efectos específicos sobre las caídas presidenciales.

De otro lado, algunos autores han enfatizado que la puesta en práctica de determinadas políticas económicas ha influido en las caídas presidenciales y en los conflictos políticos en América Latina (Wise et al., 2003). En específico, se ha señalado que la implantación de políticas de ajuste estructural o neoliberales aumentó la propensión de los presidentes a salir anticipadamente de sus cargos. Así lo constata Hochstetler (2006) en su evaluación cuantitativa de la estabilidad de los presidentes latinoamericanos desde 1978. En la misma línea se encuentran los hallazgos de la extensa investigación realizada para el periodo 1982-1995 por Stokes (2001) y la de Weyland (1993) al estudiar la caída del presidente Fernando Collor de Mello.

\section{Protesta social y escándalos de corrupción}

La protesta social vista como una de las variables que explica la salida anticipada de los jefes de Estado predomina en la investigación sobre caídas presidenciales. Esto se verifica tanto en los estudios realizados desde la ciencia política (Kim \& Bahry, 2008; Pérez-Liñán, 2003, 2007, 2014; Hochstetler, 2006; Lucero, 2001; García, 2000; Abente, 1999; Weyland, 1993) como en los que provienen de otras disciplinas. ${ }^{2} \mathrm{Al}$ respecto, quienes otorgan un papel estelar a la protesta social señalan que la presencia de movilización ciudadana es decisiva para el resultado final de un proceso de inestabilidad institucional

2 Se puede consultar la producción de la revista osal publicada por el Consejo Latinoamericano de Ciencias Sociales (CLACSO) en http://www.clacso.org.ar/institucional/1h3.php. Para Ecuador, y concentrados en el análisis del cesamiento en sus funciones del presidente Mahuad, véanse Ciriza (2000), Barrera (2000), Ramírez (2000), Ospina (2000) y Dávalos (2000). Un análisis de la protesta social y su incidencia en los episodios de inestabilidad en Argentina se encuentra en Scribano \& Schuster (2001) y Lozano (2001). La caída (fugaz) del presidente Chávez encuentra un análisis coyuntural en López Maya (2003). Hay un buen análisis de la destitución del presidente Sánchez de Lozada en Mamani (2003). 
orientado a obtener la salida anticipada de los presidentes (Hochstetler \& Edwards, 2009; Hochstetler, 2006).

En esta tradición de estudios se argumenta que la protesta social ha estado presente en todos los casos en los que se ha llegado a la remoción de los presidentes de América Latina (Hochstetler, 2006: p. 403). El rol decisivo de la movilización popular también se observa en las caídas presidenciales suscitadas en África, aunque con un impacto menor que en América Latina (Kim \& Bahry, 2008: p. 817). No obstante, existe un debate en torno a si la protesta social cumple un papel esencial en dichos fenómenos o si, por el contrario, es en las negociaciones entre las élites donde surge la decisión política de terminar antes de tiempo el periodo de un presidente. En esta última situación, la protesta social ocuparía un espacio subalterno, constituyéndose así en un mecanismo de presión que subyace a los acuerdos alcanzados por los actores políticos (Carey, 2003a, 2003b; Schamis, 2002).

La literatura especializada también ha afirmado que los escándalos de corrupción constituyen una variable exógena que actúa como un poderoso disparador de la salida anticipada de los presidentes (Hochstetler \& Edwards, 2009; Kim \& Bahry, 2008; Pérez-Liñán, 2007; Hochstetler, 2006; Mainwaring \& Pérez-Liñán, 2005; Valenzuela, 2004; Kada, 2003; Weyland, 1993). En ese plano, hay constatación empírica de que los escándalos de corrupción influyen poderosamente sobre los legisladores de cara a iniciar juicios políticos - formales o ad hoc — en contra de los presidentes (Kim, 2013; Hochstetler, 2006; Baumgartner, 2003). No obstante, los hallazgos empíricos en sentido contrario no dejan de estar presentes. Al respecto, Kim \& Bahry (2008: p. 816) señalan que los escándalos de corrupción que involucran directamente a los presidentes generan menos riesgo de una salida anticipada que lo que la literatura especializada asume.

\section{Una propuesta teórica para el análisis de las caídas presidenciales}

Como se desprende de nuestra revisión, los estudios sobre las caídas presidenciales no están exentos de tensiones en cuanto a las variables que explican las salidas anticipadas de los presidentes. Aun cuando muchos concuerdan en el papel de los factores políticos, económicos y sociales, la discrepancia aparece cuando se pretende identificar la variable que desencadena la caída de los presidentes. Un argumento opuesto indicaría que el fenómeno que se estudia es multicausal y que, por tanto, no es factible desagregar las variables explicativas hasta identificar un orden secuencial. No obstante, lo que en este artículo argumentamos, en concordancia con Hochstetler (2006), Pérez-Liñán (2007, 2014), Aversa (2013) y Polga-Hecimovich \& Basabe (2015), es que es posible 
establecer una serie de eventos que, entrelazados entre sí, permiten explicar de forma analítica la presencia o no de caídas presidenciales.

La idea central que proponemos es que el análisis de las caídas presidenciales y sus explicaciones se relaciona con lo que metodológicamente se conoce como complejidad causal (Braumoeller, 2003; Bennett \& George, 1997). De los dos tipos de complejidad causal, conocidos también como cadenas causales, el que más se adapta al estudio de las caídas presidenciales es el que Braumoeller (2003) denomina causalidad coyuntural múltiple (multiple conjunctural causation). La lógica que articula este tipo de causalidad señala que existen situaciones en las que varias causas interactúan para producir efectos y que la forma de esa interacción es descrita por el operador lógico "y" (Ragin, 1987: pp. 89-93). En términos formales, la causalidad coyuntural múltiple indica que $X_{1}$ y $X_{2}$ y $X_{3}$ producen $Y$. El segundo tipo de complejidad causal, que opera bajo la lógica de lo sustituible (substitutability), se diferencia en el sentido de que recurre al operador "o". La formalización de este planteamiento sería que $X_{1} \circ X_{2} \circ X_{3}$ producen $Y$ (Cioffi-Revilla \& Starr, 1995; Most \& Starr, 1984). ${ }^{3}$

Siguiendo la lógica de la causalidad coyuntural múltiple, existen al menos dos causas $\left(\mathrm{X}_{1}\right.$ y $\left.\mathrm{X}_{2}\right)$ que sumadas provocan las caídas presidenciales $(\mathrm{Y})$. Si una de las dos causas está ausente entonces la salida anticipada del presidente no debería de darse. Nótese que la segunda causa se relaciona con la primera por lo que allí es necesario establecer un vínculo específico entre ellas. Con estas consideraciones, a continuación se evalúan las distintas variables que la literatura del tema ha señalado como influyentes en las caídas presidenciales, destacando algunas ideas clave en cuanto a su ubicación en la secuencia de eventos que desencadenan en las salidas anticipadas de los jefes de Estado.

Una primera explicación indica que las coaliciones de gobierno menos durables erosionan poco a poco el apoyo de la legislatura al ejecutivo hasta que la pugna de poderes desemboca en la salida anticipada de los presidentes. Asumir que una decisión de las élites es el factor esencial para la verificación del fenómeno que nos interesa evaluar implica defender la idea de que la toma de decisiones políticas se encuentra reservada para estos jugadores de veto (Tsebelis, 2002). De hecho, la literatura indica que uno de los cambios observados en la política, al menos en América Latina, corresponde a la emergencia de nuevos

3 Otras formas de complejidad causal constituyen el análisis de contextos: $\mathrm{X}_{2}$ produce $\mathrm{Y}$ pero solo en la presencia de $\mathrm{X}_{1}$ (Goertz, 1994); el de condiciones necesarias y suficientes, en que $\mathrm{X}_{1}$ y $\mathrm{X}_{2}$ producen $\mathrm{Y}$, o $\mathrm{X}_{1}$ o $\mathrm{X}_{2}$ producen $\mathrm{Y}$ (Braumoeller \& Goertz, 2000; Dion, 1998); y el de condiciones INUs (an insufficient but necessary part of a condition which is itself unnecessary but sufficient for the result): $\left(\mathrm{X}_{1} \mathrm{y}_{2}\right)$ o $\left(\mathrm{X}_{3}\right.$ y $\left.\mathrm{X}_{4}\right)$ producen $\mathrm{Y}$ (Mackie, 1965: p. 245). 
actores sociales que por medio de diversas formas de acción colectiva influyen cada vez más en la arena de lo público. Por tanto, proponer que la protesta social surge a posteriori de cuando las élites políticas deciden iniciar un proceso que desestabilice al presidente choca con la evidencia empírica hallada en América Latina y relatada en buena parte de la investigación respectiva (Kim, 2013; Hochstetler \& Edwards, 2009; Pérez-Liñán, 2007).

Los análisis sobre el grado de fragmentación del sistema de partidos políticos y el apoyo legislativo hacia el presidente siguen la lógica reseñada en el párrafo anterior. En efecto, considerar que las características de la correlación de fuerzas políticas en las arenas de decisión formales constituyen el punto clave que desencadena las salidas anticipadas de los presidentes implica pensar en sociedades sin mayor ampliación de la esfera de participación ciudadana. Por otro lado, la explicación relacionada con el bajo apoyo popular al presidente cuando este inicia su periodo de gobierno tampoco tiene suficiente fundamento. Si esta fuera la variable clave para explicar la salida anticipada de los presidentes, la inestabilidad presidencial sería la norma en la región. Además, el porcentaje de votos que reciben los presidentes en primera vuelta tiene que ver tanto con la elección de los votantes como con la influencia de los diseños institucionales sobre dicha decisión.

Otras explicaciones señalan que el desempeño de la economía es la variable dirimente para explicar las caídas presidenciales. El argumento que se plantea señala que cuando los indicadores económicos no marchan bien en un país, el descontento de diferentes sectores aumentará de tal modo que, si la crisis es demasiado aguda, se incrementan las probabilidades de que el presidente salga prematuramente de su cargo. A diferencia de otros factores que cita la literatura, la variable económica impacta directamente en el bienestar cotidiano de los ciudadanos. Así, aun cuando la corrupción pública y los escándalos políticos influyan indirecta o directamente en la opinión de la ciudadanía respecto al gobierno, este efecto casi siempre es menor al que produce una crisis económica. Como corolario, si hay estabilidad económica en el corto plazo, incluso con escándalos de corrupción en las más altas esferas de gobierno o deficientes rendimientos de las instituciones democráticas, disminuyen las probabilidades de que un proceso de inestabilidad concluya con la salida del presidente.

Para tornar operativo el concepto de crisis económica lo hemos homologado con lo que la literatura considera como crisis financiera. En este sentido, las definiciones varían en función de los parámetros utilizados para la medición. Claessens \& Kose (2013) identifican cuatro tipos de crisis financieras: de divisas, de interrupción brusca de los flujos de capital, de deuda (interna o externa), y bancaria. Los dos primeros se conceptualizan con base en criterios estricta- 
mente cuantitativos mientras que los restantes se verifican por medio de una valoración cualitativa. Para nuestro análisis asumimos la crisis de divisas como un referente esencial que da cuenta de la conmoción económica que atraviesa un país. Siguiendo a Reinhart \& Rogoff (2009), consideramos que habrá crisis de divisas en cualquier contexto en el que la tasa de inflación anual supere el $20 \%$. Aunque los cuatro tipos de crisis pueden ocurrir de forma aislada o concurrente — como en Ecuador en 1999 y principios de 2000—, en este artículo sostenemos que cualquiera de ellos tiene la capacidad de desencadenar una salida presidencial anticipada.

Bajo la conceptualización otorgada a la crisis económica, resultan menos importantes las políticas que los gobiernos apliquen pues allí solo se encuentran los medios que el presidente ha utilizado para resolver problemas económicos, y lo que interesa a la población son los resultados en el corto plazo. Así, cuando un país soporta una crisis económica de grandes magnitudes, independientemente de que provenga de un programa de ajuste estructural, de un modelo neokeynesiano o de una política económica basada en una fuerte intervención del Estado, aumentarían las probabilidades de que se desate un proceso de inestabilidad presidencial. Aunque el vínculo entre desempeńos económicos e inestabilidad política se asemeja a la propuesta teórica de Przeworski et al. (2000), lo que aquí sostenemos es que las crisis económicas pueden llevar a la salida de los presidentes pero no al rompimiento del régimen democrático, que es la principal hipótesis de los autores mencionados.

De otro lado, los escándalos de corrupción han sido mencionados como decisivos en las salidas presidenciales. A fin de articular un argumento lógico que coloque a esta variable como el "disparador" de aquellas se debería asumir que las sociedades latinoamericanas valoran ampliamente el manejo transparente de los recursos públicos y que, por tanto, existe menor permisibilidad a la corrupción. En dicho escenario, la reacción social a un escándalo de corrupción podría llegar al extremo de requerir la salida del presidente. Desafortunadamente, la mayoría de países de América Latina son de aquellos en los que un acto de corrupción lejos de ser sancionado moral o éticamente es asumido como parte de los códigos de comportamiento aceptados socialmente (Pepys, 2007). Por ello, aunque las denuncias y escándalos de corrupción son parte del escenario que rodea a los casos de caídas presidenciales, no hay argumentos lo suficientemente fuertes para creer que ese sea el "disparador" de un proceso de inestabilidad presidencial que concluya en el cambio del jefe de Estado.

Finalmente, las protestas ciudadanas que terminan con la salida anticipada de los presidentes se darían, en realidad, por un motivo específico anterior a tal movilización social. En otras palabras, si la economía se maneja sin proble- 
mas, hay mediano respeto por las instituciones democráticas y el gobierno no afronta graves situaciones de corrupción, las demandas ciudadanas difícilmente tendrían como pretensión final la sustitución del presidente. En consecuencia, nuestra propuesta es que aunque la movilización ciudadana actúa como mecanismo para procesar demandas suscitadas en diversas esferas, solo cuando el evento se inscribe en la arena económica el descontento popular puede concluir en la salida adelantada de los presidentes. Acorde a la idea de causalidad coyuntural múltiple argumentamos que para que se verifique una salida presidencial anticipada $(\mathrm{Y})$ se requiere la existencia de una crisis económica $\left(\mathrm{X}_{1}\right)$ "y" la movilización de protesta social en las calles $\left(\mathrm{X}_{2}\right)$. De lo dicho surgen los siguientes corolarios:

a) En ausencia de crisis económica $\left(\mathrm{X}_{1}\right)$ y aun con movilización de protesta social en las calles $\left(\mathrm{X}_{2}\right)$, no se concluye en caída presidencial $(\mathrm{Y}){ }^{4}$

b) En presencia de crisis económica $\left(\mathrm{X}_{1}\right)$, pero sin movilización de protesta social en las calles $\left(\mathrm{X}_{2}\right)$, no se concluye en caída presidencial $(\mathrm{Y})$.

Aunque las crisis económicas y la movilización de protesta social en las calles $\left(\mathrm{X}_{1} \mathrm{y}_{2}\right)$ dan cuenta de caídas presidenciales $(\mathrm{Y})$, esto no excluye la aparición de cualquiera de las otras variables que la literatura especializada ha identificado. Sin embargo, todos estos factores políticos, económicos o sociales son contextuales y podrían estar o no presentes. A diferencia de lo que sugiere un análisis cuantitativo en el que se identifican las variables presentes y su peso específico en las caídas presidenciales, el marco teórico que aquí esbozamos permite capturar la cadena de eventos causales que, articulados entre sí, provocan que un presidente salga antes de concluir su cargo. Además, el análisis de causalidad coyuntural múltiple permite evidenciar que las crisis económicas representan el disparador de la inestabilidad que termina con la salida anticipada de los mandatarios. No obstante, en países que carecen de una ampliación de la ciudadanía observable en la organización popular y las protestas en las calles, aun cuando haya deficientes desempeños económicos los presidentes se mantendrían en funciones.

Analíticamente, una vez que se produce una crisis económica o que la economía termina en un escenario de crisis, las protestas sociales que previamente se han mantenido vivas, aunque no con la suficiente intensidad y frecuencia, presionan al presidente para que realice cambios en la conducción del gobier-

4 A partir del análisis de contextos (Goertz, 1994) la lógica en este corolario sería: la movilización de protesta social en las calles $\left(\mathrm{X}_{2}\right)$ concluye con la caída presidencial $(\mathrm{Y})$ solo si previamente está presente una crisis económica $\left(\mathrm{X}_{1}\right)$.

138 - S. Basabe-Serrano, J. Polga-Hecimovich | Desempeño económico y protesta ciudadana como detonantes de las caídas presidenciales: el caso ecuatoriano | Perfiles Latinoamericanos, 25(50) | Flacso México | pp. 129-153 DOI: $10.18504 / \mathrm{pl} 2550-007-2017$ 
no. A la movilización ciudadana por razones económicas en ocasiones se pueden unir otra clase de demandas, entre las que estarían las relacionadas con el irrespeto a las instituciones democráticas, violaciones a las libertades civiles o escándalos de corrupción gubernamental. En conjunto, aunque partiendo de la fortaleza de los reclamos por el desempeño de la economía, la movilización en las calles presiona de distintas formas hasta llegar a requerir la salida del presidente. Como ya se ha dicho, el hecho de que la crisis económica se haya dado por la implementación de políticas de ajuste estructural u otras menos ortodoxas es irrelevante.

A fin de precisar el tipo de protesta ciudadana a la que nos referimos en este artículo, utilizamos el concepto de movilización social heterogénea de Pérez-Liñán (2007) retomado por Ollier (2008) para discutir la inestabilidad presidencial argentina. En efecto, cuando conceptualizamos la movilización social tenemos la idea de que se encuentre conformada por una coalición de sectores medios y populares y que su repertorio de demandas se dirija de forma explícita contra el presidente (Ollier, 2008; Pérez-Liñán, 2007). Esta puntualización es fundamental pues en ausencia de los sectores sociales anotados, la protesta ciudadana puede originarse como consecuencia de crisis económicas, pero no concluir con la destitución del presidente. Los episodios de conflictividad afrontados por la presidenta argentina Cristina Fernández darían cuenta de protestas ciudadanas en las que no hay movilización social heterogénea y que, por tanto, no derivaron en su salida anticipada (Ollier, 2013, 2014).

Ante un escenario de convulsión y caos como el que sucede cuando a la crisis económica sigue una movilización social heterogénea, los actores políticos cercanos al gobierno no tienen mayor capacidad de negociación a fin de evitar la salida anticipada del presidente. Si el partido oficialista no tiene mayoría legislativa, si las coaliciones de gobierno son frágiles, o si ambas condiciones se dan, la decisión de las élites políticas de apoyar la caída presidencial es la opción que genera menores costos ante la ciudadanía. Así, la existencia de mayor o menor fragmentación partidista en ese momento resulta irrelevante pues la coyuntura es tal que, independientemente de la correlación de fuerzas políticas, la caída presidencial es solo una cuestión de tiempo.

De otro lado, y aun si la coalición de gobierno es estable y fuerte - algo poco probable en un escenario de crisis económica-, la presión de las protestas sociales conduce a que la salida prematura presidencial sea lo que menor costo político ocasiona al partido oficialista. Adicionalmente, el porcentaje de votos con el que el presidente inició su gestión, así como el tamaño de su bancada legislativa también serían irrelevantes en el escenario descrito. Una vez que los detonantes económico y de movilización ciudadana se han puesto en marcha, cualquiera de los factores identificados por la literatura terminan siendo con- 
tingentes. Aun cuando las variables que la investigación previa ha señalado podrían incidir en las caídas presidenciales, sostenemos que una crisis económica sumada a la protesta social en las calles constituye la fórmula que explica los eventos de inestabilidad presidencial que este artículo analiza. ${ }^{5}$

Cabe anotar además que la fortaleza de la movilización ciudadana está influida por el rol que juega la oposición política de cara a incrementar o no el desgaste de la figura presidencial. Aunque la protesta social es la variable más importante que sigue a la crisis económica, los actores políticos de oposición que promueven acciones directas en contra del presidente facilitan que la movilización en las calles sea la variable desencadenante para la inestabilidad presidencial. Un referente empírico de esto lo constituyen las acciones de dirigentes peronistas orientadas a inflamar las movilizaciones ciudadanas que concluyeron con la salida anticipada del presidente Fernando de la Rúa (Auyero, 2007).

\section{El caso ecuatoriano: una aproximación empírica}

Para verificar empíricamente la propuesta teórica previa, en esta parte analizamos el caso ecuatoriano, uno de los más interesantes en la región por su alta inestabilidad en términos de caídas presidenciales (Mejía \& Polga-Hecimovich, 2011; Pérez-Liñán, 2007). Comparamos dos gobiernos, uno que completó su periodo (Durán-Ballén) y otro en el que el presidente salió anticipadamente del cargo (Mahuad). De esta forma ofrecemos varianza en la variable dependiente y a la par controlamos por algunas de las variables citadas en la literatura especializada. Consideramos fuentes primarias para la medición de algunas variables y recurrimos a datos ya existentes para otras. Los periodos analizados van de agosto de 1992 a agosto de 1996 (gobierno de Durán-Ballén) y de agosto de 1998 a enero de 2000 (gobierno de Mahuad).

Primero hay que señalar que los gobiernos de Durán-Ballén y Mahuad corresponden a corrientes ideológicas similares, representadas en el Partido Unidad Republicana (PUR) —una disidencia del Partido Social Cristiano (PSC) - y en la Democracia Popular (DP), respectivamente. Ambos partidos — que por su tendencia se les puede calificar de centro derecha- compartían la idea de que eran necesarias políticas de ajuste estructural. A la par, tanto Durán-Ballén como Mahuad tuvieron un apoyo minoritario en las urnas para alcanzar el poder. El primero obtuvo $31.87 \%$ en la primera vuelta electoral mientras que el

5 No obstante, hay casos atípicos a nuestra propuesta teórica. Las caídas de los presidentes Zelaya en Honduras y Lugo en Paraguay son dos eventos que se dieron a pesar de la ausencia de crisis económicas.

140 - S. Basabe-Serrano, J. Polga-Hecimovich | Desempeño económico y protesta ciudadana como detonantes de las caídas presidenciales: el caso ecuatoriano | Perfiles Latinoamericanos, 25(50) | Flacso México | pp. 129-153 DOI: $10.18504 / \mathrm{pl} 2550-007-2017$ 
segundo alcanzó 34.91\%. En el balotaje, Durán-Ballén ganó la presidencia con $57.32 \%$ de votos y Mahuad lo hizo con $51.15 \%$. Si los valores de la primera vuelta revelan la real intención de voto de la ciudadanía por los candidatos, la información empírica da cuenta de la ausencia de mayores diferencias entre un presidente y otro.

La debilidad del apoyo electoral a estos dos presidentes se reflejó también en las bancadas de los partidos oficialistas. Durán-Ballén llegó al poder con $16.66 \%$ de asientos legislativos y Mahuad con el $26.45 \% .{ }^{6} \mathrm{Al}$ mismo tiempo, el número de partidos fue elevado en una y otra legislaturas. En el gobierno del PUR se registraron doce agrupaciones políticas y en la administración de la DP hubo diez partidos con representación en el Congreso Nacional. Este dato se relaciona con el grado de fragmentación partidista relativamente alto que se dio en las dos legislaturas. Acorde con el índice de Laakso \& Taagepera (1979), diseñado para Ecuador por Pachano (2011), dicho valor corresponde a 0.78 en el gobierno de Durán-Ballén y a 0.76 en el de Mahuad. Aunque el tiempo de duración de las coaliciones sí muestra diferencias entre los dos gobiernos, esto podría deberse al proceso de inestabilidad institucional que rodeó la caída de Mahuad. La tabla 1 resume los datos de la medición de las variables de entorno político que han sido discutidas.

Tabla 1. Comportamiento de las variables políticas en los gobiernos de Durán-Ballén y Mahuad

\begin{tabular}{lcc}
\hline Variable & Sixto Durán-Ballén & Jamil Mahuad \\
\hline Votación (1a. vuelta) & $31.87 \%$ & $34.91 \%$ \\
Bancada oficialista & $16.66 \%$ & $26.45 \%$ \\
Partidos en legislatura & 12 & 10 \\
Fragmentación & 0.78 & 0.76 \\
Coaliciones legislativas & agosto 1992-agosto 1994 & agosto1998-febrero 1999 \\
& (PUR, PSC, PCE) & (DP, PSC, FRA, PCE) \\
& agosto 1994-julio 1995 & marzo 1999-junio 1999 \\
& (PUR, PSC, PCE) & (DP, ID, PCK) \\
& octubre 1995-agosto 1996 & \\
\hline
\end{tabular}

Fuentes: Basabe-Serrano \& Polga-Hecimovich (2013), Pachano (2011), Mejía \& Polga-Hecimovich (2011).

En cuanto a escándalos de corrupción, si bien durante los dos gobiernos se dieron situaciones que comprometieron la figura presidencial, ninguna tuvo la trascendencia para poner en riesgo la permanencia del jefe de Estado. El índi-

6 El porcentaje de asientos legislativos oficialistas en el periodo del presidente Durán-Ballén se refiere solo a la bancada del pur. No se considera la representación legislativa del Partido Conservador Ecuatoriano (PCE) del entonces vicepresidente Alberto Dahik. 
ce de percepción de la corrupción de Transparencia Internacional en Ecuador para 1996 —el primer año en que este país se incorporó a la medición— fue de 3.19, y en 1998 y 1999 fue de 2.3 y 2.4, respectivamente. Aunque en el gobierno de Mahuad este índice descendió, denotando una mayor corrupción en la esfera pública, la evaluación cualitativa de los casos de más trascendencia no marca diferencias ostensibles. A partir de una exhaustiva revisión del Diario Hoy, uno de los matutinos más importantes del país y con acceso vía Internet para los dos periodos presidenciales analizados, no se detectó una variación considerable entre los escándalos de corrupción de uno y otro gobiernos. La tabla 2 describe el análisis de la variable anotada.

Tabla 2. Escándalos de corrupción en los gobiernos de Durán-Ballén y Mahuad

\begin{tabular}{ll}
\hline Gobierno & Casos de corrupción \\
\hline Sixto Durán-Ballén & "Flores y Miel", "Gastos reservados", "General Núñez", "Comité de Información y Contacto \\
& $\begin{array}{l}\text { Externo", "La Fabril" } \\
\text { Jamil Mahuad }\end{array}$ \\
& "Depósito de Aspiazu a campaña electoral", "Uso de avión de uso oficial por parte de Ministro \\
& Rivadeneira", "Feriado bancario"
\end{tabular}

Fuente: Diario Hoy (1992-1996, 1998-2000).

En el gobierno de Durán-Ballén el caso de corrupción más sonado fue el de "Gastos reservados", en el que el principal implicado fue el vicepresidente Alberto Dahik. Dicho escándalo tuvo relación con el destino ilegítimo de recursos públicos que, siendo destinados a la seguridad nacional, se utilizaron - entre otras cosas - para obtener votos en la legislatura en temas que el gobierno consideraba fundamentales. El escándalo llegó al extremo de que el vicepresidente fue enjuiciado políticamente por el Congreso Nacional aunque no se obtuvieron los votos para su destitución-, se dictó una orden de prisión preventiva en su contra y finalmente renunció a su cargo. A pesar de este desenlace, la permanencia de Durán-Ballén nunca estuvo en entredicho. Así, el gobierno de este último se inscribe en lo que Hochstetler (2006) señala como una amenaza a la estabilidad presidencial, pero sin la salida anticipada del jefe de Estado.

En el caso de Mahuad, la opinión pública ha considerado que el denominado "Feriado bancario" y el subsiguiente "Congelamiento de fondos", son los casos de mayor corrupción. Ambas decisiones han recibido lecturas diversas. Por un lado, quienes fueron parte de ese gobierno consideran que se trataron de actos gubernamentales que, más allá de los efectos que pudieron ocasionar a la economía nacional, no es factible que se los asimile como hechos de corrupción. Por otro, diversos actores sociales y el exfiscal general Washington Pesántez han señalado que los decretos ejecutivos que contienen las decisiones 
ya relatadas configuraron el delito de peculado. ${ }^{7}$ Más allá de la discusión jurídica al respecto, lo que interesa enfatizar aquí es que ambas decisiones se dieron en marzo de 1999, es decir, diez meses antes de la salida anticipada de Mahuad (Conaghan, 2012). Por ello, aunque este podría ser un factor interviniente, no se lo puede asimilar como el detonante de la caída presidencial.

Acorde con nuestra propuesta teórica, se debe observar que las diferencias específicas entre los dos gobiernos se encuentran en el desempeño de la economía. Para el análisis hemos tomado como referente empírico la variación en la tasa de inflación durante los dos periodos presidenciales y asumimos que se trata de una crisis financiera si dicha tasa supera el 20\% (Reinhart \& Rogoff, 2009). Como se advierte en la figura 1 , mientras que en el gobierno de DuránBallén el mes de mayor índice inflacionario fue el de octubre de 1992 (65.86\%), para el de Mahuad este indicador llega al 78.07\% en enero de 2000, precisamente cuando se verifica la caída del presidente.

En general, tras los primeros meses del gobierno de Durán-Ballén, en los que la tasa de inflación fue alta, con el paso del tiempo este indicador decreció, concluyendo el mandato presidencial en $25.17 \%$ para agosto de 1996 . Por el contrario, el gobierno de Mahuad presenta una tendencia al alza durante toda su gestión, sobre todo a raíz de los ya comentados "Feriado bancario" y "Congelamiento de fondos". De hecho, entre febrero y marzo de 1999 la tasa de inflación se disparó en casi 15 puntos porcentuales.

Figura 1. Inflación mensual, 1992-2000

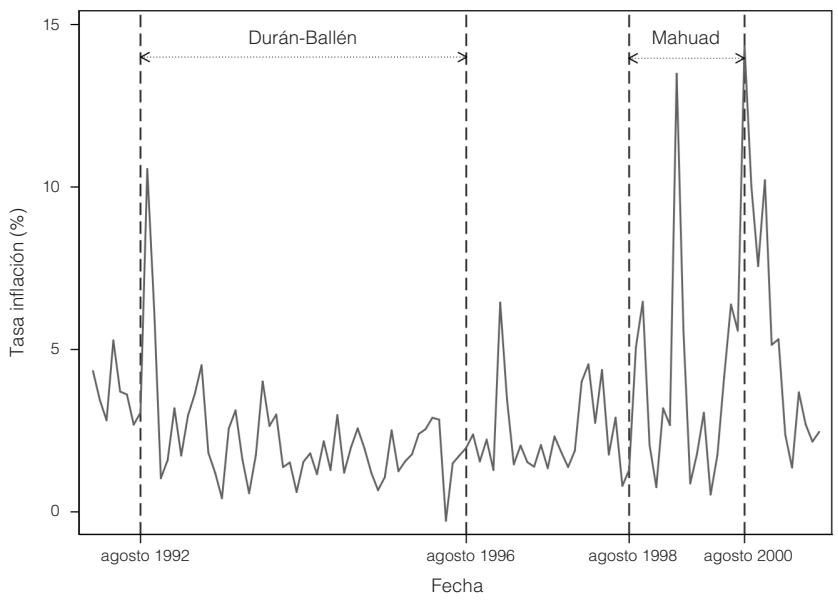

Fuente: Elaboración propia con datos mensuales del Instituto Nacional de Estadísticas y Censos (INEC).

7 “Fiscalía acusa a ex presidente Mahuad por crisis financiera de 1999”, Diario Hoy, 22 de octubre de 2008. 
Las diferencias en cuanto al manejo de la economía ya descritas no guardan relación con las políticas implementadas por ambos gobiernos pues, como señalamos en párrafos previos, las dos administraciones se orientaron por la idea de que era necesario implementar un programa económico de ajuste estructural. En otras palabras, y a pesar de la casi nula aplicación de políticas orientadas hacia el mercado en el caso ecuatoriano (Lora \& Panizza, 2002), uno y otro gobiernos pueden ser calificados como neoliberales. La tabla 3 resume las leyes aprobadas cuya direccionalidad era una política de ajuste estructural.

Tabla 3. Políticas de ajuste estructural en los gobiernos de Durán-Ballén y Mahuad

\begin{tabular}{|c|c|c|}
\hline Ley & $\begin{array}{l}\text { Estatus } \\
\text { actual }\end{array}$ & $\begin{array}{l}\text { Fecha de } \\
\text { publicación }\end{array}$ \\
\hline \multicolumn{3}{|l|}{ DURÁN-BALLÉN } \\
\hline Ley Especial de Telecomunicaciones & Reformada & 10 -ago-92 \\
\hline Ley de Presupuestos del Sector Público & Derogada & 30-nov-92 \\
\hline Ley de Mercado de Valores & Vigente & 28-may-93 \\
\hline Ley de Transformación y Rehabilitación de la Empresa Ecuatoriana de Aviación & Vigente & 9-nov-93 \\
\hline Reforma a la Ley de Hidrocarburos & Vigente & 29-nov-93 \\
\hline Ley de Modernización del Estado & Reformada & 31-dic-93 \\
\hline Reforma a la Ley de Régimen Tributario & Vigente & $31-$ dic-93 \\
\hline Ley General de Instituciones del Sistema Financiero & Vigente & 12-may-94 \\
\hline Reforma a la Ley de Zonas Francas & Vigente & $15-j u n-94$ \\
\hline Ley de Creación del Fondo de Solidaridad & Vigente & 24-mar-95 \\
\hline Reforma a la Ley de Modernización del Estado & Reformada & 11-may-95 \\
\hline Reforma a la Ley Especial de Telecomunicaciones & Reformada & 30-ago-95 \\
\hline Reforma a la Ley de Régimen Tributario Interno & Vigente & 31-ago-95 \\
\hline Reforma a la Ley de Modernización del Estado & Reformada & 29-dic-95 \\
\hline Reforma a la Ley de Contratación Pública & Vigente & 10-jun-96 \\
\hline $\begin{array}{l}\text { Reforma a la Ley de Protección a los Representantes, Agentes o Distribuidores de } \\
\text { Empresas Extranjeras }\end{array}$ & Derogada & 5 -jul-96 \\
\hline Reforma a la Ley General de Instituciones del Sistema Financiero y Otras & Derogada & $31-j u l-96$ \\
\hline \multicolumn{3}{|l|}{ MAHUAD } \\
\hline Reforma a la Ley de Hidrocarburos & Reformada & 26 -ago-98 \\
\hline Reforma a la Ley General de Instituciones del Sistema Financiero & Vigente & 7-sep-98 \\
\hline Reforma a la Ley de Régimen del Sector Eléctrico & Vigente & 30-sep-98 \\
\hline Ley de Reordenamiento en Materia Económica, en el Área tributario-Financiera & Reformada & 1-dic-98 \\
\hline Reforma a la Ley de Zonas Francas & Vigente & 16-mar-99 \\
\hline Reforma a la Ley de Comercio Exterior e Inversiones & Derogada & 25-mar-99 \\
\hline Ley para la Reforma de las Finanzas Públicas & Reformada & $30-a b r-99$ \\
\hline Reforma a la Ley General de Instituciones del Sistema Financiero y Otras & Vigente & 13-may-99 \\
\hline Reforma a la Ley de Mercado de Valores & Vigente & 1-jul-99 \\
\hline Ley de Racionalización Tributaria & Derogada & \\
\hline Reforma a la Ley General de Instituciones del Sistema Financiero & Vigente & \\
\hline
\end{tabular}

Fuente: Registro Oficial del Ecuador y base electrónica LEXIS para los periodos 1992-1996 y 1998-1999.

144 S. Basabe-Serrano, J. Polga-Hecimovich | Desempeño económico y protesta ciudadana como detonantes de las caídas presidenciales: el caso ecuatoriano | Perfiles Latinoamericanos, 25(50) | FLACso México | pp. 129-153

DOI: $10.18504 / \mathrm{pl} 2550-007-2017$ 
La escalada inflacionaria iniciada en Ecuador a fines de 1999 y principios de 2000 constituye entonces el factor que desencadena una serie de protestas sociales que se van incrementando en cuanto a intensidad y frecuencia hasta llegar a la demanda ciudadana por la salida de Mahuad. Como consta en la figura 2, elaborada con el reporte trimestral sobre conflictividad social de Ecuador Debate, la cantidad de protestas en los dos periodos de gobierno muestra diferencias importantes. En la administración de Durán-Ballén, septiembre de 1995 es el mes de mayor conflictividad, con 59 movilizaciones, mientras que en la de Mahuad, julio de 1999 es el más convulsionado, con 95 protestas sociales. Al final de su gobierno, en agosto de 1996, Durán-Ballén soportó 25 episodios de conflictividad social, en tanto que la salida anticipada de Mahuad en enero de 2000 estuvo marcada por noventa protestas ciudadanas y de sectores sociales organizados.

Figura 2. Protestas sociales por mes, 1992-2000

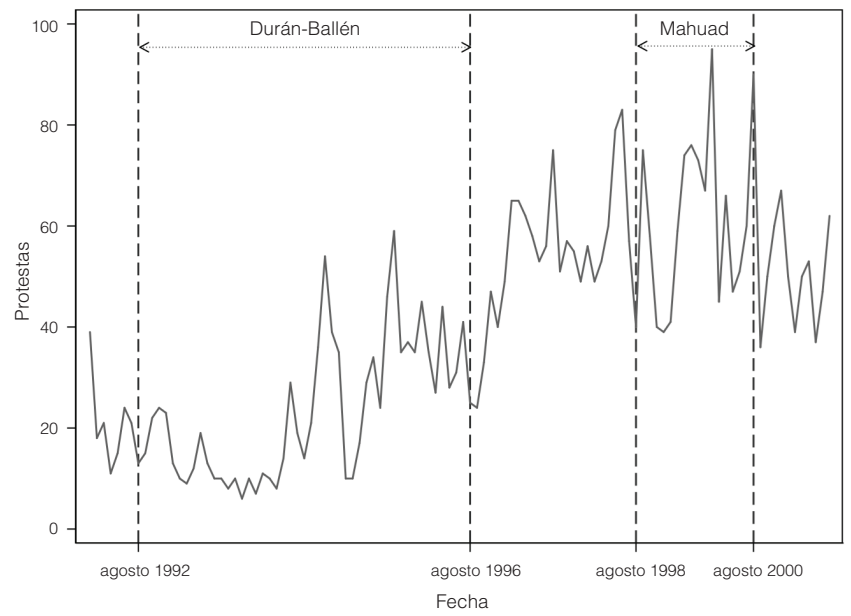

Fuente: Ecuador Debate de los años 1992-1996 y 1998-2000.

Las protestas ciudadanas observadas durante el episodio que dio lugar a la caída de Mahuad coinciden con el concepto de movilización social heterogénea que utilizamos como referente teórico en este artículo. En efecto, las marchas de protesta se focalizaron en contra del primer mandatario y no demandaban rectificaciones en el manejo del gobierno sino directamente su salida. De manera adicional, en dichas movilizaciones confluyeron tanto sectores medios como los estratos más populares de la sociedad. 
En definitiva, la crisis económica que afrontó el gobierno de Mahuad constituyó el disparador de las demandas ciudadanas que terminaron por colocar al presidente al filo de la destitución. Cuando se había llegado a este punto y el mandatario estaba prácticamente fuera del cargo, los actores políticos en la legislatura buscaron darle una salida de aparente constitucionalidad. Nótese que la actuación del Congreso Nacional se dio a posteriori de la movilización social y que esta variable fue consecuencia de la crisis económica desatada, en buena medida, como corolario de los decretos ejecutivos que dispusieron el "Feriado bancario" y el "Congelamiento de fondos" (Lucero, 2001).

El análisis previo se presta para discernir el lugar que ocupa la protesta social y la acción de las élites políticas en la caída de Mahuad. Conocer cuál es el evento previo es esencial en la discusión que ofrecemos pues de esto depende la verificación empírica de la teoría que proponemos. Hemos argumentado que la protesta social sigue a la crisis económica y no la intervención de la legislatura, pues dentro de las demandas de los sectores movilizados - maestros, sindicatos, estudiantes, indígenas, campesinos - se encontraba tanto la salida del presidente Mahuad como la de los diputados y la de los magistrados de la Corte Suprema (Diario Hoy, 2000).

Bajo dicha coyuntura, la decisión del Congreso Nacional de cesar a Mahuad debe verse como una reacción ante la posibilidad de que fueran los propios legisladores los removidos de sus cargos. Como ya se ha dicho en este artículo, independientemente de la correlación de fuerzas partidistas, cuando en un país existe crisis económica y la subsecuente movilización ciudadana en las calles, la decisión de los actores políticos de retirar el apoyo al presidente es el mecanismo que les permite asumir el menor costo posible del conflicto.

\section{Conclusiones}

En este artículo hemos discutido la literatura relacionada con las caídas presidenciales evidenciando los vacíos y la necesidad de profundizar en la investigación empírica del tema. A pesar de la investigación cuantitativa en la que se estudian muchos casos en el mundo, la ausencia de hallazgos empíricos se mantiene. Esto se debe a las perspectivas teóricas usadas por los estudiosos y a los sesgos en las mediciones realizadas. Si bien los trabajos con $n$ grande nos ayudan en cuanto a generalizaciones, la captura de información empírica de las variables explicativas se verifica a partir de aproximaciones que podrían llevar a mayores errores de medición.

Por dichas razones, en este artículo hemos argumentado que para abordar un fenómeno como el de las caídas presidenciales es necesario un esquema 
lo suficientemente analítico que permita identificar con claridad la cadena de eventos que, entrelazados entre sí, dan lugar al fenómeno que se pretende estudiar. Aplicando la noción de causalidad coyuntural múltiple, defendimos la idea de que las crisis económicas $\left(X_{l}\right)$ a las que siguen movilizaciones sociales en las calles $\left(X_{2}\right)$ explican las salidas anticipadas de los presidentes $(Y)$. Sin desconocer que otras variables citadas en la literatura especializada podrían converger en determinadas situaciones, sostenemos que la suma de crisis económica y protesta social explican de forma analítica las salidas anticipadas de los presidentes.

A fin de verificar la validez de la propuesta teórica observamos empíricamente dos casos - uno de caída presidencial y otro de mantenimiento del jefe de Estado- ocurridos en Ecuador, uno de los países más inestables de la región hasta inicios del siglo XxI. Luego del análisis quedó en evidencia la relación entre crisis económica y protesta social como variables que explican la caída del presidente Mahuad. El hecho de que buena parte de las variables señaladas por la literatura se encuentran controladas en los dos episodios comparados otorga mayor fiabilidad a los resultados empíricos obtenidos. En todo caso, resta por ampliar el número de observaciones de tal modo que permita proponer generalizaciones. En tal sentido, la principal contribución de este artículo se da en el plano teórico pues innova los trabajos hasta ahora desarrollados por la ciencia política.

Respecto a la necesidad de incrementar los casos analizados, el trabajo de Ollier (2008), sobre la inestabilidad presidencial argentina entre 2001 y 2003, llega a conclusiones similares a las que hemos expuesto, aunque desde una aproximación metodológica diferente. Para Ollier, cuando jefes de Estado débiles, en términos materiales o simbólicos, deben asumir un escenario económico conflictivo se sientan las bases para la inestabilidad presidencial. A pesar de que el énfasis de Ollier no solo está en la influencia de la movilización social como desencadenante de la salida de los presidentes, su propuesta coincide con la nuestra en cuanto a que el punto de partida de un proceso de inestabilidad es la crisis económica del país.

Para una futura agenda de investigación de las caídas presidenciales no solo será necesario aumentar el $n$, sino además mejorar la calidad de la evidencia empírica que sustenta la investigación, sobre todo la de naturaleza cuantitativa. Lo dicho coloca a la investigación en la encrucijada de ganar en generalización a costa de mediciones menos precisas o disminuir en el alcance de las conclusiones a beneficio de reducir los sesgos entre los conceptos y los referentes empíricos. En dicha dicotomía - difícil de resolver- no se encuentran puntos intermedios en la literatura especializada. Por tanto, planteamos que el diseño de investigaciones en las que se compaginen estrategias cuantitativas y cualitativas puede ayudar a resolver las tensiones empíricas que todavía permanecen 
en el análisis de las caídas presidenciales. Lejos de ser una temática con hallazgos firmes y conclusiones de consenso, el estudio de aquellas tiene aún mucho camino por recorrer.

\section{Referencias}

Abente-Brun, D. (1999). People Power' in Paraguay. Journal of Democracy, 10(3), 93-100.

Auyero, J. (2007). La zona gris. Violencia colectiva y política partidaria en la Argentina contemporánea. Buenos Aires: Siglo XXI Editores.

Aversa, C. I. (2013). La dinámica ejecutivo-legislativo en la resolución de las crisis presidenciales latinoamericanas. Buenos Aires: Facultad de Ciencias Sociales y Relaciones InternacionalesPontificia Universidad Católica Argentina.

Barrera, A. (2000). Ecuador, o cuando la crisis se hace cotidiana. Reflexiones sobre los episodios del 21 y 22 de enero de 2000. OSAL, Observatorio Social de América Latina, (1), 11-16.

Basabe-Serrano, S. \& Polga-Hecimovich, J. (2013). Legislative Coalitions and Judicial Turnover under Political Uncertainty: The Case of Ecuador. Political Research Quarterly, 66(1), 154-166.

Baumgartner, J. C. (2003). Introduction: Comparative Presidential Impeachment. En Baumgartner, J. \& Kada, N. (Eds.). Checking Executive Power: Presidential Impeachment in Comparative Perspective. Westport, CT: Praeger.

Bennett, A. \& George, A. (1997). Research Design Tasks in Case Study Methods. En MacArthur Foundation Workshop on Case Study Methods. Belfer Center for Sciences and International Affairs (вCSIA). Harvard University.

Braumoeller, B. (2003). Causal Complexity and the Study of Politics. Political Analysis, 11(3), 209-233.

Braumoeller, B. \& Goertz, G. (2000). The Methodology of Necessary Conditions. American Journal of Political Science 44(4), 844-858.

Carey, J. M. (2003a). Transparency vs. Collective Action: Fujimori's Legacy and the Peruvian Congress. Comparative Political Studies, (36), 983-1006.

Carey, J. M. (2003b). Presidentialism and Representative Institutions. En Domínguez, J. \& Shifter, M. (Eds.). Constructing Democratic Governance in Latin America (2a. ed.). Baltimore: The Johns Hopkins University Press. 
Cheibub, J. A. (2002). Minority Governments, Dead-lock Situations, and the Survival of Presidential Democracies. Comparative Political Studies, 35(3), 284-312.

Cioffi-Revilla, C. y Starr, H. (1995). Opportunity, Willingness and Political Uncertainty: Theoretical Foundations of Politics. Journal of Theoretical Politics, (7), 447-476.

Ciriza, A. (2000). 21 de enero del 2000 ¿Golpe de estado o revuelta india en el Ecuador? OSAL, Observatorio Social de América Latina, 1(1), 6-10.

Claessens, S. \& Kose, M. A. (2013). Financial Crises: Explanations, Types, and Implications. IMF Working Paper. Washington, DC: International Monetary Fund.

Conaghan, C. (2012). Prosecuting Presidents: The Politics within Ecuador's Corruption Cases. Journal of Latin American Studies, 44(4), 649-678.

Cox, G. \& Morgenstern, S. (2002). Epilogue: Latin America's Reactive Assemblies and Proactive Presidents. En Morgenstern, S. \& Nacif, B. (Eds.). Legislative Politics in Latin America. Nueva York: Cambridge University Press.

Dávalos, P. (2000). Las transformaciones políticas del movimiento indígena ecuatoriano. OSAL, Observatorio Social de América Latina, (1), 25-29.

Diario Hoy. (2000, 19 de enero). Mahuad instó a un acuerdo nacional.

Diario Hoy. (2008, 22 de octubre). Fiscalía acusa a ex presidente Mahuad por crisis financiera de 1999.

Dion, D. (1998). Evidence and Inference in the Comparative Case Study. Comparative Politics, (30), 127-145.

García Calderón, E. (2000). High Anxiety in the Andes: Peru’s Decade of Living Dangerously. Journal of Democracy, (12), 46-58.

Goertz, G. (1994). Contexts of International Politics. Cambridge: Cambridge University Press.

Haggard, S. \& Kaufman, R. R. (1995). The Political Economy of Democratic Transitions. Princeton, New Jersey: Princeton University Press.

Hochstetler, K. (2006). Rethinking Presidentialism: Challenges and Presidential Falls in South America. Comparative Politics, 38(4), 401-418.

Hochstetler, K. \& Edwards, M. E. (2009). Failed Presidencies: Identifying and Explaining a South America Anomaly. Journal of Politics in Latin America, 1(2), 31-57. 
Instituto Nacional de Estadísticas y Censos. Recuperado de http://www.ecuadorencifras.gob.ec/ institucional/home/

Jones, M. P. (1995). Electoral Laws and the Survival of Presidential Democracies. Notre Dame, Indiana: University of Notre Dame Press.

Kada, N. (2003). The Role of Investigative Committees in the Presidential Impeachment Processes in Brazil and Colombia. Legislative Studies Quarterly, (28), 29-54.

Kim Hun, Y.. \& Bahry, D. (2008). Interrupted Presidencies in Third Wave Democracies. The Journal of Politics, $70(3), 807-822$.

Kim Hun, Y. (2013). Impeachment and Presidential Politics in New Democracies. Democratization, 20(1), 1-35.

Laakso, M. \& Taagepera, R. (1979). The "Effective" Number of Parties: A Measure with Application to Western Europe. Comparative Political Studies, 12(1), 3-28.

LEXIs. Recuperado de http://www.lexis.com.ec/website/default.aspx

Linz, J. (1990). The Perils of Presidentialism. Journal of Democracy, 1(1), 51-69.

López Maya, M. (2003). Venezuela en la encrucijada. OSAL, Observatorio Social de América Latina, $\operatorname{III}(9), 55-60$.

Lora, E. A. \& Panizza, U. (2002). Structural Reforms in Latin America under Scrutiny. InterAmerican Development Bank, Research Department. Working Paper 470.

Lozano, C. (2001). Contexto económico y político en la protesta social de la Argentina contemporánea. OSAL, Observatorio Social de América Latina, (5), 156-157.

Lucero, J. A. (2001). High Anxiety in the Andes: Crisis and Contention in Ecuador. Journal of Democracy, (12), 59-73.

Mackie, J. L. (1965). Causes and Conditions. American Philosophical Quarterly, (2), 245-264.

Mainwaring, S. (1993). Presidentialism, Multipartism, and Democracy-the Difficult Combination. Comparative Political Studies, 26(2), 198-228.

Mainwaring, S. \& Pérez-Liñán, A. (2005). Latin American Democratization since 1978: Democratic Transitions, Break-downs, and Erosions. En Hagopian, F. \& Mainwaring, S. (Eds.). The Third Wave of Democratization in Latin America: Advances and Setbacks. Nueva York: Cambridge University Press.

150 - S. Basabe-Serrano, J. Polga-Hecimovich | Desempeño económico y protesta ciudadana como detonantes de las caídas presidenciales: el caso ecuatoriano | Perfiles Latinoamericanos, 25(50) | Flacso México | pp. 129-153 DOI: $10.18504 / \mathrm{pl} 2550-007-2017$ 
Mainwaring, S. \& Shugart M. (1997). Juan Linz, Presidentialism, and Democracy: A Critical Appraisal. Comparative Politics, 29(4), 449-472.

Mamani Ramírez, P. (2003). El rugir de la multitud: levantamiento de la ciudad de Aymara de El Alto y caída del gobierno de Sánchez Lozada. OSAL, Observatorio Social de América Latina, (12), 15-25.

Marsteintredet, L. \& Berntzen, E. (2008). Reducing the Perils of Presidentialism through Presidential Interruptions. Comparative Politics, (41), 83-101.

Mejía Acosta, A. y Polga-Hecimovich, J. (2011). Coalition Erosion and Presidential Instability in Ecuador. Latin American Politics and Society, 53(2), 87-111.

Most, B. \& Starr, H. (1984). Inquiry, Logic and International Politics. Columbia: University of South California Press.

Negretto, G. (2006). Minority Presidents and Democratic Performance in Latin America. Latin American Politics and Society, 48(3), 63-89.

Ollier, M. M. (2014). Presidencia dominante y oposición fragmentada: una construcción política. Néstor y Cristina Kirchner (2003-2011). Buenos Aires: Documentos de trabajo de la Escuela de Política y Gobierno de la Universidad Nacional de San Martín.

Ollier, M. M. (2013). La democracia invertida: entre la acefalía y la hegemonía: hacia su formulación conceptual en un estudio de caso. Revista Debates, 7(1), 199-227.

Ollier, M. M. (2008). La institucionalización democrática en el callejón: la inestabilidad presidencial en Argentina (1999-2003). América Latina Hoy, (49), 73-103.

Ospina, P. (2000). La vuelta a un día de ochenta mundos. OSAL, Observatorio Social de América Latina, (1), 30-37.

Pachano, S. (2011). Calidad de la democracia e instituciones politicas en Bolivia, Ecuador y Perú. Quito: Flacso Ecuador.

Pepys, M. N. (2007). Corruption within the Judiciary: Causes and Remedies, En Global Corruption Report 2007 (pp. 3-11). Cambridge: Cambridge University Press,

Pérez-Liñán, A. (2014). A Two-Level Theory of Presidential Instability. Latin American Politics and Society, 56(1), 34-54.

Pérez-Liñán, A. (2007). Presidential Impeachment and Democratic Accountability in Latin America, 1990-1999. Nueva York: Cambridge University Press.

S. Basabe-Serrano, J. Polga-Hecimovich | Desempeño económico y protesta ciudadana como detonantes de las caídas presidenciales: el caso ecuatoriano | Perfiles Latinoamericanos, 25(50) | Flacso México | pp. 129-153 
Pérez-Liñán, A. (2003). Presidential Crises and Democratic Accountability in Latin America, 1990-1999. En Eckstein S. E. \& Wickham-Crowley T. P. (Eds.). What Justice? Whose Justice: Fighting for Fairness in Latin America (pp. 98-132). Berkeley: Berkeley University of California Press.

Polga-Hecimovich, J. \& Basabe-Serrano S. (2015, mayo 27-31). Proceed with Caution: A Replication Study of Pérez-Liñán's Theory of Presidential Impeachment and Removal. Presentada durante el xxxiII Congreso de la Asociación de Estudios Latinoamericanos (LASA). San Juan, Puerto Rico.

Przeworski, A., Álvarez, M., Cheibub, J. A. \& Limongi, F. (2000). Democracy and Development: Political Institutions and Economic Performance, 1950-1999. Cambridge: Cambridge University Press.

Ragin, C. (1987). The Comparative Method: Moving Beyond Qualitative and Quantitative Strategies. Berkeley: Berkeley University of California Press.

Ramírez Gallegos, F. (2000). El 21 de enero del 2000. OSAL, Observatorio Social de América Latina, (1), 17-21.

Registro Oficial. Recuperado de https://www.registroficial.gob.ec

Reinhart, C. M. \& Rogoff, K. S. (2009). This Time Is Different: Eight Centuries of Financial Folly. Princeton: Princeton University Press.

Schamis, H. (2002). Argentina: Crisis and Democratic Consolidation. Journal of Democracy, 13(2), 81-94.

Scribano, A. \& Schuster, F. (2001). Protesta social en la Argentina de 2001: entre la normalidad y la ruptura. OSAL, Observatorio Social de América Latina, (5), 17-22.

Stokes, S. C. (2001). Mandates and Democracy: Neoliberalism by Surprise in Latin America. Cambridge: Cambridge University Press.

Tsebelis, G. (2002). Veto Players: How Political Institutions Work. Princeton, New Jersey: Princeton University Press.

Valenzuela, A. (2004). Latin American Presidencies Interrumpted. Journal of Democracy, 15(4), 5-19.

Weyland, K. (1993). The Rise and Fall of President Collor and its Impact on Brazilian Democracy. Journal of Interamerican Studies and World Affairs, 35(1), 1-37. 
Wise, C., Roett, R. \& Paz, G. (2003). Post-Stabilization Politics in Latin America: Competition, Transition, Collapse. Washington, DC: Brookings Institution.

Recibido el 1 de mayo de 2015. Aceptado el 17 de diciembre de 2015. 